\title{
Plasmonically Enhanced ZnO Thin-Film-Photo-Transistor with Dynamic Responsivity Control
}

\author{
Ayşe Özcan ${ }^{1, *}$, Enes Battal ${ }^{1,2}$, Fatih Bilge Atar ${ }^{1,2}$ and Ali Kemal Okyay ${ }^{1,2}$ \\ ${ }^{1}$ UNAM - Institute of Materials Science and Nanotechnology, Bilkent University, TR-06800, Ankara, Turkey \\ ${ }^{2}$ Bilkent University, Department of Electrical and Electronics Engineering, Bilkent, TR-06800, Ankara, Turkey
}

*Tel: +90 (312) 290 1557, e-mail: ayse.ozcan@bilkent.edu.tr

\begin{abstract}
We fabricated an $\mathrm{ZnO}$ based thin-film phototransistor with electrically tunable photo-responsivity operating in the UV and visible spectra and designed plasmonic structures enhancing the device performance up to 6 folds below the band-gap of $\mathrm{ZnO}$.
\end{abstract}

$$
\text { I. INTRODUCTION }
$$

Recently, Zinc-Oxide $(\mathrm{ZnO})$ based thin film transistors (TFTs) attracted much attention due to their potential of replacing amorphous Silicon (a-Si) which is used widely for conventional TFTs [1]. Especially, higher mobility of $\mathrm{ZnO}$ compared to a-Si makes it more plausible for TFT applications [2]. Several techniques are used to deposit $\mathrm{ZnO}$ channel layer of transistors including RF magnetron sputtering [3], chemical vapor deposition [4], molecular beam epitaxy (MBE) [5], laser ablation [6] and atomic layer deposition (ALD) [7] techniques. Among these techniques, ALD comes forward with high quality film growth, high-precision thickness control and large area application advantages [7].

Along with its supreme electrical properties, $\mathrm{ZnO}$ has been started to use as an active layer for optoelectronic applications. $\mathrm{ZnO}$ has a strong absorption up to the wavelength of about $375 \mathrm{~nm}$ [8] due to its band-gap of $3.31 \mathrm{eV}$. However, ALD grown $\mathrm{ZnO}$ has considerable amount of traps that give rise absorption in the visible spectrum observed in the previous photoluminescence studies [8]. $\mathrm{ZnO}$ based light emitting diodes[9], photo-detectors [4], solar cells [10] and phototransistors [11] have been realized in this spectrum. Yet, the performance of such devices has been limited to bulk absorption properties of $\mathrm{ZnO}$ layers.

In recent years, many methods have been developed to improve the performance of photodetectors and photo-voltaic devices. Surface plasmons, collective oscillations of electrons coupled with electromagnetic waves, have been proven to be very useful at ultra-violet and visible spectrum for increasing device absorption [12]. In order to maximize absorption, localized and propagating surface plasmons excited by nanoparticles or gratings are very useful due to their immense subwavelength scaling properties.

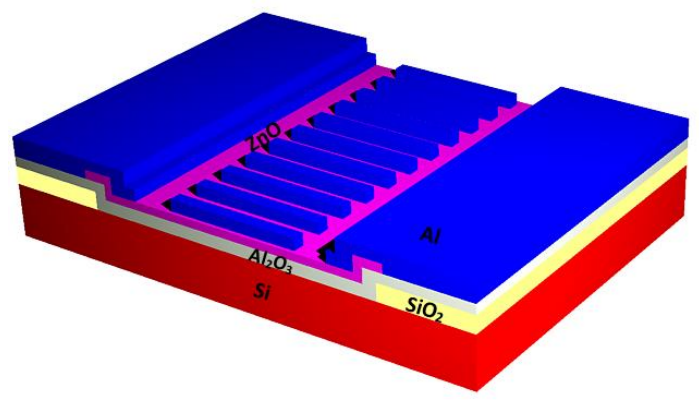

Figure 1 a) 3-dimensional depiction of the plasmonically enhanced $\mathrm{ZnO}$ based photo-transistor device structure

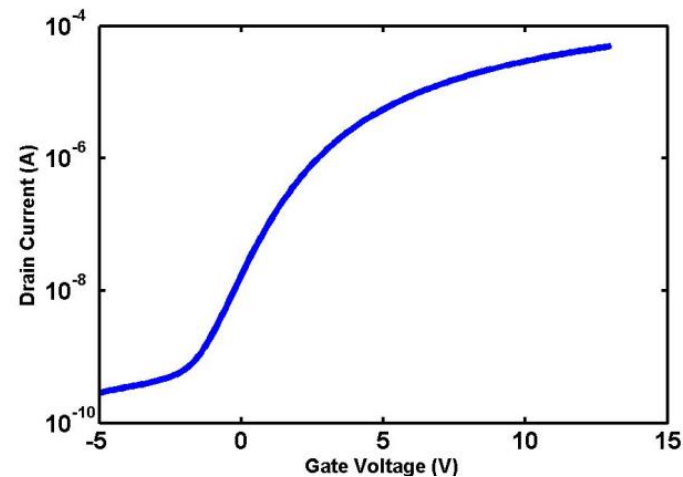

Figure 2 - At $0.5 \mathrm{~V}$ of drain to source voltage $\left(\mathrm{V}_{\mathrm{DS}}\right)$, contrast ratio of about $10^{4}$ and turn on voltage of $-2 \mathrm{~V}$ is observed in drain-source current to gate voltage $\left(I_{D S}-V_{G S}\right)$ relation of the fabricated device.

In this work, we demonstrate a plasmonically enhanced $\mathrm{ZnO}$ based thin film photo-transistor (TFPT). We fabricated and characterized an ALD grown $\mathrm{ZnO}$ based TFPT operating at ultraviolet and visible wavelengths. We electrically characterized transistor characteristics of the fabricated device and we performed optical responsivity measurements. We designed metallic gratings enhancing overall photo-response of the fabricated device up to 6 fold in the wavelength range of $400-600 \mathrm{~nm}$

\section{EXPERIMENT}

The device fabrication by deposition of a $\mathrm{SiO} 2$ contact isolation layer with plasma enhanced chemical vapor deposition on a p-type highly doped $\mathrm{Si}$ substrate. This layer is patterned with optical lithography and etched with buffered oxide etchant (BOE). Then, an $\mathrm{A} 12 \mathrm{O} 3$ gate oxide layer is deposited at $250^{\circ} \mathrm{C}$ for 200 cycles with Cambridge Savannah 100 ALD System using tri-methyl-aluminum and water precursor. A 100 cycles of $\mathrm{ZnO}$ channel layer is deposited at $250^{\circ} \mathrm{C}$ using diethyl-zinc and water. The $\mathrm{ZnO}$ layer is patterned with optical lithography and etched with dilute $\mathrm{H}_{2} \mathrm{SO}_{4}$ solution. After an optical lithography process, a 100nm thick Al drain and source layer is deposited followed by lift-off. The finalized structure is depicted in Figure 1.

The fabricated transistors are electrically characterized by Keithley 4200-SCS semiconductor parameter analyzer system. The drain-source current to gate voltage $\left(I_{D S} V_{G S}\right)$ is plotted in Figure 2 for $0.5 \mathrm{~V}$ drain to source $\left(V_{D S}\right)$ bias. The turn on gate voltage, $\mathrm{V}_{\mathrm{ON}}$ and the $\mathrm{ON} / \mathrm{OFF}$ ratio, $I_{O N} / I_{O F F}$ are determined to be $-2 \mathrm{~V}$ and $10^{4}$, respectively. Threshold voltage of this device is extracted from the $\sqrt{I_{D S}}-V_{G S}$ relation as $1 \mathrm{~V}$. From the $\log I_{D S^{-}}$ $V_{G S}$ curve, the sub-threshold slope of the device is determined to be $1.17 \mathrm{~V} / \mathrm{dec}$. 
Under a modulated illumination with a mechanical chopper at a frequency of about $375 \mathrm{~Hz}$ and a lock-in amplifier, we measured the responsivity, defined as ratio of the photocurrent

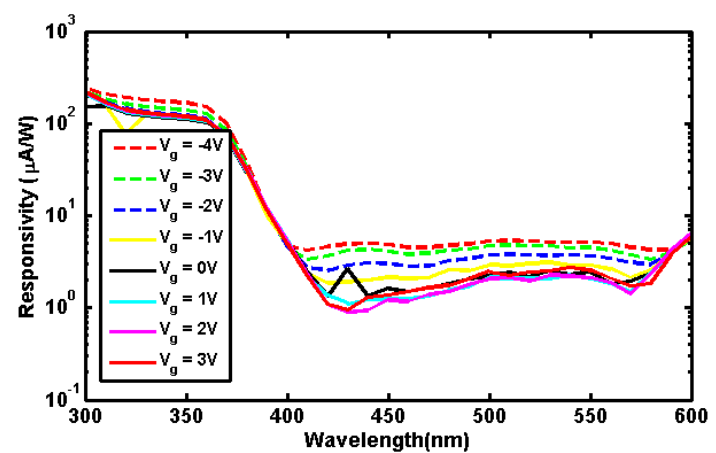

Figure 3 - The responsivity of the device is controlled by applying different gate bias voltages. The responsivity for the region below the band-gap is much lower than that of the region above band-gap.

$\left(I_{D S}\right)$ to incident optical power. Figure 3 shows the responsivity of the photo-transistor under different gate bias voltages. Applying reverse bias from the gate depletes the trapped carriers and allows more absorption in the visible region. In this way, the photo-response from the photo-transistor can be controlled by applying different gate-bias. Yet, the responsivity in the spectral region below the band-gap remains low comparing to the region above band-gap.

\section{Simulation RESUlTS}

In order to increase the responsivity of the device in the visible region, we integrate rectangular plasmonic structures on top of the active $\mathrm{ZnO}$ layer as shown in Figure 1. We performed 2-dimensional finite difference time domain simulations using FDTD solutions from Lumerical Inc. The rectangular gratings are assumed to be placed periodically. The height of the rectangular grating is kept constant at $100 \mathrm{~nm}$, whereas, width (w) and distance (d) parameters are swept for optimization. The refractive index of $\mathrm{ZnO}$ and $\mathrm{Al} 2 \mathrm{O} 3$ layers are obtained using a spectroscopic ellipsometer (JaWoollam VVase). For $\mathrm{Al}$ and Si layers, optical constants from literature data [] are used. We compared the absorption inside the active $\mathrm{ZnO}$ layer for the case with and without plasmonic gratings assuming a light polarization suitable for plasmon excitation i.e. electric field vector is normal to the gratings. We also investigated the effect of metallic gratings, experimentally.

In Figure $4 \mathrm{a}$, overall absorption enhancement integrated over $400-600 \mathrm{~nm}$ spectrum is depicted. The optimum structure parameters are found to be $\mathrm{w}=90 \mathrm{~nm}$ and $\mathrm{d}=125 \mathrm{~nm}$ with an overall enhancement of about 7 times. Spectral absorption enhancement for the optimum structure is depicted in Figure $4 \mathrm{~b}$. The highest enhancement is observed at the wavelength where the plasmon resonance is much stronger near the
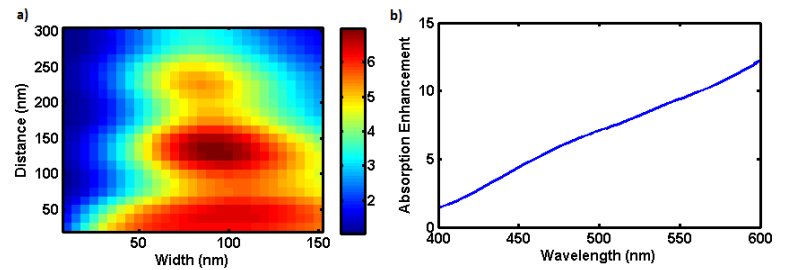

Figure 4 - a) Dependence of overall enhancement for 400-600nm spectrum on the structure parameters, distance (d) and width (w). b) For the optimum structure, spectral absorption enhancement is depicted
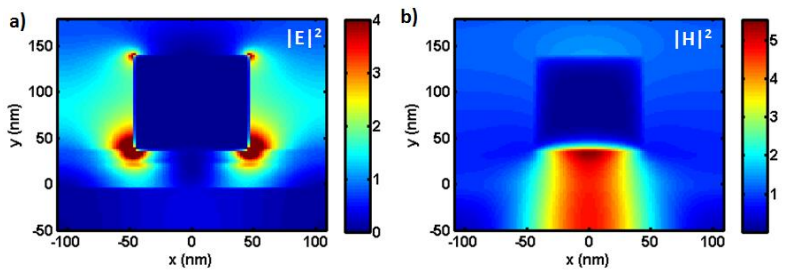

Figure 5 - Electric and magnetic field intensity profiles at the wavelength of highest absorption ehnancement (600nm)

$\mathrm{ZnO} / \mathrm{Al}$ interface as can be seen from the electric field intensity profile in Figure 5a. Absorption enhancement is attributed to localized surface plasmon (LSP) resonance excited at the $\mathrm{ZnO} / \mathrm{Al}$ interface which is weakly coupled to the LSP mode excited at air/Al interface. $\mathrm{H}$ field intensity profile in Figure $5 \mathrm{~b}$ indicates that the plasmonic structure acts like a strong forward scatterer.

\section{CONCLUSION}

We fabricated an ALD grown $\mathrm{ZnO}$ based thin film phototransistor (TFPT) and designed plasmonic grating structure exhibiting overall performance enhancements up to 6 fold in the overall spectrum of $400-600 \mathrm{~nm}$. We predicted that the responsivity in the spectral region below the band-gap can be increased up to the values of the responsivity in the region above band-gap. This study paves the way of realizing efficient photo-transistors operating in the UV and visible region.

\section{ACKNOWLEDGEMENTS}

This work was supported in part by European Union Framework Program 7 Marie Curie IRG Grant 239444, COST NanoTP, TUBITAK Grants 109E044, 112M004 and 112E052. The authors acknowledge support from TUBITAK-BIDEB.

\section{REFERENCES}

[1] R. L. Hoffman, B. J. Norris, and J. F. Wager, Applied Physics Letters, vol. 82, pp. 733-735, 2003.

[2] E. Fortunato, P. Barquinha, and R. Martins, Advanced Materials, vol. 24, pp. 2945-2986, 2012.

[3] P. F. Carcia, R. S. McLean, M. H. Reilly, and J. G. Nunes, Applied Physics Letters, vol. 82, pp. 1117-1119, 2003.

[4] Y. Liu, C. R. Gorla, S. Liang, N. Emanetoglu, Y. Lu, H. Shen, et al., Journal of Elec Materi, vol. 29, pp. 69-74, 2000/01/01 2000.

[5] Y. Chen, D. M. Bagnall, H.-j. Koh, K.-t. Park, K. Hiraga, Z. Zhu, et al., Journal of Applied Physics, vol. 84, pp. 3912-3918, 1998.

[6] M. Hiramatsu, K. Imaeda, N. Horio, and M. Nawata, Journal of Vacuum Science \& Technology A: Vacuum, Surfaces, and Films, vol. 16, pp. 669-673, 1998

[7] S. Lee, Y. Im, and Y.-B. Hahn, Korean J. Chem. Eng., vol. 22, pp. 334-338, 2005/03/01 2005.

[8] L. E. Aygun, F. B. Oruc, F. B. Atar, and A. K. Okyay, Photonics Journal, IEEE, vol. 5, pp. 2200707-2200707, 2013.

[9] Y. Ryu, T.-S. Lee, J. A. Lubguban, H. W. White, B.-J. Kim, Y.-S. Park, et al., Applied Physics Letters, vol. 88, p. 241108, 2006.

[10] I. Repins, M. A. Contreras, B. Egaas, C. DeHart, J. Scharf, C. L. Perkins, et al., Progress in Photovoltaics: Research and Applications, vol. 16, pp. 235239, 2008.

[11] H. S. Bae, M. H. Yoon, J. H. Kim, and S. Im, Applied Physics Letters, vol. 83 , pp. 5313-5315, 2003

[12] D. K. Gramotnev and S. I. Bozhevolnyi, Nat Photon, vol. 4, pp. 83-91, 2010 